\title{
Gastrointestinal disorders next to respiratory infections as leading symptoms of X-linked agammaglobulinemia in children - 34-year experience of a single center
}

\author{
Malgorzata Pac' ${ }^{1}$ Ewa A. Bernatowska ${ }^{1}$, Jarosław Kierkuś², Józef P. Ryżko², \\ Joanna Cielecka-Kuszyk ${ }^{3}$, Teresa Jackowska ${ }^{4}$, Bożena Mikołuć ${ }^{5}$
}

\begin{abstract}
'Department of Immunology, The Children's Memorial Health Institute, Warsaw, Poland

2Department of Gastroenterology, Hepatology and Nutrition Disorders, The Children's Memorial Health Institute, Warsaw, Poland

${ }^{3}$ Department of Pathology, The Children's Memorial Health Institute, Warsaw, Poland ${ }^{4}$ Department of Pediatrics, Medical Center of Postgraduate Education, Department of Pediatrics, Bielański Hospital, Warsaw, Poland

${ }^{5}$ Department of Pediatrics, Medical University, Bialystok, Poland
\end{abstract}

Submitted: 20 January 2015

Accepted: 19 April 2015

Arch Med Sci 2017; 13, 2: 412-417

DOI: https://doi.org/10.5114/aoms.2016.60338

Copyright $\odot 2016$ Termedia \& Banach

\section{Abstract}

Introduction: Respiratory tract infections constitute the most frequent manifestation of X-linked agammaglobulinemia (XLA). There are not many papers elucidating gastrointestinal (GI) disorders, including inflammatory bowel disease (IBD), in such patients. The aim of the study was to evaluate the occurrence of gastrointestinal disorders and IBD compared to respiratory tract infections in XLA individuals.

Material and methods: Of 1563 patients with primary immunodeficiencies diagnosed in the Department of Immunology, the Children's Memorial Health Institute (CMHI), 66 boys had a provisional diagnosis of agammaglobulinemia. Forty-four subjects fulfilled definitive ESID (European Society for Immunodeficiencies) diagnostic criteria of XLA. A retrospective analysis of medical history of XLA patients was undertaken.

Results: Recurrent respiratory tract infections, particularly bronchitis (73\%) and pneumonia (59\%), were the most common symptoms of XLA. The GI disorders constituted the next main manifestation (63.6\%), followed by upper respiratory tract infections. Twenty-six of 28 XLA patients with GI disorders complained of diarrhea, which was resolved generally after immunoglobulin therapy introduction. Single but prolonged episodes of Campylobacter jejuni diarrhea were reported in two individuals. Inflammatory bowel disease of mild to moderate activity was diagnosed in 1 patient, and local enteritis of mild activity in another one.

Conclusions: Gastrointestinal disorders were one of the main manifestations of XLA, reported almost as often as lower respiratory tract infections. The most common Gl symptom was diarrhea, which usually resolved after immunoglobulin therapy was started. Infections caused by Giardia lamblia were reported occasionally. Inflammatory bowel disease was diagnosed quite exceptionally, which presumably may be connected with normal T cell immunity.

Key words: primary antibody deficiency, inflammatory bowel disease, respiratory infections, children.

\author{
Corresponding author: \\ Malgorzata Pac \\ Department of Immunology \\ The Children's Memorial \\ Health Institute \\ Av. Dzieci Polskich 20 \\ 04-730 Warsaw, Poland \\ Phone: +48 2281573 78, \\ +48604448855 \\ Fax: +48 228151839 \\ E-mail: malgorzata.pac@ \\ wp.pl
}




\section{Introduction}

$\mathrm{X}$-linked agammaglobulinemia (XLA) is a rare inherited disease associated with mutation in the Btk gene located on the long arm of the $X$ chromosome and coding for Bruton tyrosine kinase. This protein is strongly involved in intramedullary differentiation of B lymphocytes, the arrest of which leads to significantly diminished antibody production [1-4]. The most frequent manifestations of XLA are recurrent or prolonged respiratory tract infections (RTI). Patients are highly susceptible to infections of lungs, bronchi, sinuses, and ears with encapsulated bacteria (Streptococcus pneumoniae, Haemophilus influenzae), complicated by chronic lung disease (mostly bronchiectases) and chronic rhinosinusitis (CRS). Also Giardia and enterovirus infection of the gut are reported as common. In contrast to other primary antibody deficiencies such as common variable immunodeficiency (CVID), XLA is less often associated with a variety of autoimmune conditions, probably because of the normal cell-mediated immune response [4-7].

The aim of the study was to evaluate the occurrence of gastrointestinal disorders, including inflammatory bowel disease, in comparison with respiratory tract infections in children with XLA under the care of one center.

\section{Material and methods}

One thousand five hundred sixty-three cases of primary immunodeficiencies (PID) were diagnosed in the Department of Immunology, the Children's Memorial Health Institute in Warsaw between 1980 and 2014. Among them 66 patients met definitive, possible or probable criteria of Bruton's agammaglobulinemia (www.esid.org). Retrospective analysis of medical charts of 44 selected boys with a definitive diagnosis of XLA, including demographic, clinical and laboratory data, was undertaken. All patients were seen routinely every 1-4 months with general and immunological assessment (i.e. ESR, hematology, biochemistry, CRP, immunoglobulin level, and as needed: immunophenotyping of lymphocytes, functional lymphocyte tests). Due to current problems, additional investigations were recommended, i.e. computed tomography (CT) scan, bacteriological swabs, cultures, spirometry. Molecular analyses of the Btk gene were performed courtesy of the J.J. van Dongen group, Erasmus MC, Rotterdam.

Due to the observational nature of the study, statistic analysis was not applicable.

\section{Results}

Sixty-six boys out of 1563 PID patients diagnosed in the Department of Immunology met ESID definitive, probable or possible criteria of XLA. In 44 of them XLA was established definitively (www.esid.org). Demographic and basic immunological laboratory data at diagnosis are shown in Table I. Patients presented very low levels of all three main immunoglobulin isotypes at diagnosis with the mean level of IgG $=1.33 \mathrm{~g} / \mathrm{l}$ (range: 0-4.0 g/l), $\mathrm{lgA}=0.18 \mathrm{~g} / \mathrm{l}$ (range: $0-1.34 \mathrm{~g} / \mathrm{l}$ ) and $\lg M=0.23 \mathrm{~g} / \mathrm{l}$ (range: $0-1.23 \mathrm{~g} / \mathrm{l}$ ). The total number of $B$ cells was decreased and varied between 0 and 391 cells/ $\mu$ l, with a mean value of 24.8 cells/ $\mu \mathrm{l}$ and $0.65 \%$. Numbers and percentage of CD3+ cells, as well as CD4+ and CD8+ cells, were within the normal range for age (data not shown in this article). First symptoms of the disease occurred at a mean age of 13 months (range: 1 month 7 years). Before the diagnosis lower respiratory tract infections were of the highest frequency: $73 \%$ of patients suffered from bronchitis, over half of them from pneumonia (Table II). Replacement therapy was introduced at the age of 3 years and 9 months on average. The immunoglobulin treatment included intravenous infusions with a standard dose of $0.4-0.6 \mathrm{~g} / \mathrm{kg}$ every 3 or 4 weeks, modified according to clinical results. Within the next years in some patients the route of immunoglobulin administration was changed from intravenous to subcutaneous (with one quarter of the monthly dose once a week). After the introduction of immunoglobulin replacement therapy, particularly subcutaneously, LRTI were seen less frequently (data published earlier) [8]. Chronic lung disease (CLD), mostly bronchiectases, was present in 12 boys. Seven of them developed CLD before diagnosis, while 5 did so during immunoglobulin therapy. Upper respiratory tract infections (URTI) were reported in $43 \%$ of the analyzed group. Otitis and sinusitis were counted separately, and every fourth boy suffered from otitis. Sinusitis was reported in 15 (34\%) individuals, chronic rhinosinusitis in 7. In the majority of cases, S. pneumoniae and $H$. influenzae were isolated from the nose, throat, or sputum. Other clinical manifestations, mostly of an infectious origin, such as meningitis, skin infections and abscesses, were observed less commonly. Six boys had a history of sepsis before the diagnosis and immunoglobulin treatment. In 2 of them pneumococcal origin was documented in the medical records. Another one experienced two episodes of meningitis and sepsis in a short time - the first one caused by Neisseria meningitidis and after 3 months $S$. pneumoniae. Gastrointestinal symptoms were observed in 28 (63.6\%) boys, forming the second largest group of symptoms. Diarrhea was the most common reason for visits to the doctor in 26 patients (Table III). The vast majority of patients (21 of 26) experienced recurrent episodes of diarrhea before the 
Table I. Immunological and demographic data of 44 XLA patients

\begin{tabular}{|lcccc|}
\hline Variable & Mean & Maximum & Minimum & SD \\
\hline $\operatorname{lgG}[\mathrm{g} / \mathrm{l}]$ & 1.33 & 4.74 & 0.0 & 1.29 \\
\hline $\operatorname{lgA}[\mathrm{g} / \mathrm{l}]$ & 0.18 & 1.34 & 0.0 & 0.27 \\
\hline $\operatorname{lgM}[\mathrm{g} / \mathrm{l}]$ & 0.23 & 1.23 & 0.0 & 0.57 \\
\hline B cells [\%] & 0.65 & 9.0 & 0.0 & 1.78 \\
\hline B cells [cells/ul] & 24.8 & 391 & 0 & 93.2 \\
\hline Age at diagnosis [months] & 47 & $120(10$ years $)$ & $12(1$ year $)$ & 40 \\
\hline Onset of symptoms [months] & 13 & 84 & 1 & 23 \\
\hline Replacement therapy [months] & 47 & 120 & 12 & 40 \\
\hline
\end{tabular}

Table II. Clinical manifestation of 44 XLA patients

\begin{tabular}{|lcc|}
\hline Clinical manifestation & $\begin{array}{c}\text { Number } \\
\text { of patients }\end{array}$ & $\begin{array}{c}\text { Percent of all } \\
\text { XLA patients }\end{array}$ \\
\hline Bronchitis & 32 & 73 \\
\hline Pneumonia & 26 & 59 \\
\hline URTI - other & 19 & 43 \\
\hline Otitis & 18 & 41 \\
\hline Sinusitis & 15 & 34 \\
\hline Chronic lung disease & 12 & 27 \\
\hline $\begin{array}{l}\text { Gastrointestinal } \\
\text { symptoms }\end{array}$ & 28 & 63.6 \\
\hline Sepsis & 6 & 14 \\
\hline $\begin{array}{l}\text { Meningitis/ } \\
\text { encephalitis }\end{array}$ & 6 & 16 \\
\hline Arthritis & 6 & 4.5 \\
\hline Abscesses & 6 & 14 \\
\hline Deaths & 6 & 14 \\
\hline
\end{tabular}

diagnosis of XLA. Problems were resolved when intravenous immunoglobulin (IVIg) therapy was introduced. Sporadic cases of acute diarrhea were reported in 5 patients. Most patients suffered from diarrhea of unknown origin. Surprisingly, only 1 boy had established G. lamblia infection. In individual patients viruses as well as Escherichia coli and S. typhimurium were identified.

Two boys had single but prolonged episodes of diarrhea due to Campylobacter jejuni which occurred after recognition of XLA and on immunoglobulin therapy. One boy was on intravenous immunoglobulin therapy, while the second one was on subcutaneous, with satisfactory IgG trough levels. Both of them were successfully treated with antibiotics. The first one reported abdominal pain and weight loss at the same time, which disappeared after treatment. The second one had an episode of norovirus diarrhea before diagnosis
Table III. Gastrointestinal symptoms in 44 XLA patients

\begin{tabular}{|c|c|c|}
\hline Symptom & $\begin{array}{c}\text { Number } \\
\text { of patients }\end{array}$ & $\begin{array}{c}\text { Percent of } \\
\text { XLA patients }\end{array}$ \\
\hline Diarrhea: & 26 & 59 \\
\hline Recurrent episodes & 21 & 48 \\
\hline Sporadic episodes & 5 & 11 \\
\hline G. lamblia & 1 & 2.2 \\
\hline Rotavirus & 1 & 2.2 \\
\hline C. jejuni & 2 & 4.4 \\
\hline E. coli & 1 & 2.2 \\
\hline $\begin{array}{l}\text { Ascarias } \\
\text { lumbricoides }\end{array}$ & 1 & 2.2 \\
\hline Salmonella & 1 & 2.2 \\
\hline Other viruses & 1 & 2.2 \\
\hline Weight loss & 5 & 11.4 \\
\hline Abdominal pain & 8 & 18.1 \\
\hline $\begin{array}{l}\text { Gastroscopic } \\
\text { evaluation }\end{array}$ & 6 & 13.6 \\
\hline $\begin{array}{l}\text { Colonoscopic } \\
\text { evaluation }\end{array}$ & 4 & 9 \\
\hline
\end{tabular}

of XLA and several episodes of vomiting and diarrhea after, with negative additional tests including stool examination (culture, parasites, etc.). The suspicion of lactose intolerance was aroused when improvement on a lactose-free diet was observed.

Eight boys suffered from abdominal pain and 5 from weight loss. Both symptoms were observed in 3 individuals, including 1 boy with $C$. jejuni diarrhea and a good outcome following antibiotic therapy. Another boy, in addition to abdominal pain and weight loss, presented recurrent pneumonia and bronchitis, complicated by bronchiectases (before diagnosis and immunoglobulin treatment). 
Several additional tests and consultations (endocrine, hematologic, bacteriologic, etc.) to explain short stature and low weight, as well as anemia, were undertaken without definitive diagnosis. Finally panenodoscopic evaluation revealed no macroscopic changes and only chronic active local enteritis and gastritis (cecum, rectum, antrum) of low intensity with no indication to treat it.

Three other boys underwent endoscopic diagnostics (either gastroscopy or colonoscopy or both) due to abdominal pain in 2 individuals, and abdominal pain together with weight loss in the other one. In the first 2 boys neither macroscopic nor histological signs of any pathology was found. The third, with late diagnosis of XLA (aged approximately 7 years), was found to have IBD. As a teenager he had abdominal pain without diarrhea, weight loss (below $3^{\text {rd }}$ percentile), chronic anemia, elevated inflammation markers, low IgG trough levels and no diarrhea. Concomitant intestinal (Salmonella, Shigella, Campylobacter, Clostridium, ova, cysts, and parasites) and other infections were excluded. The attempt to increase the dose and change the route of immunoglobulin administration failed. Colonoscopy showed ulcerations and aphthae in the terminal ileum with histological signs of chronic inflammation of the large bowel (transverse and sigmoid colon) of mild to moderate activity together with crypt microabscesses in the sigmoid colon (Figure 1).

A very similar clinical course was observed in a teenager with agammaglobulinemia with an unknown gene defect, positive family history, and very low immunoglobulin levels and B cell numbers at diagnosis, in whom a mutation in the BTK gene was not found. The only difference was previous $C$. jejuni infection and chronic diarrhea. Both patients were treated with sulphasalazine alone or together with corticosteroids at diagnosis. However, following exacerbations the therapy had to be modified, and azathioprine was added, with a good response. No need for biological treatment or surgery was observed.

\section{Discussion}

A wide spectrum of infectious and non-infectious manifestations is described in patients with primary antibody deficiencies (PAD). Regardless of the type of immunodeficiency, recurrent airway, ear, nose and throat (ENT) infections are the most frequently occurring symptoms [2, 9]. Antibody-deficient patients, especially agammaglobulinemic and CVID, have an increased risk of developing blood-borne bacterial infections, particularly prior to the diagnosis and institution of adequate therapy with gamma globulin $[10,11]$.

The PAD patients frequently suffer from gastrointestinal complications, with chronic or recurrent diarrhea sometimes accompanied by malabsorption and weight loss. In CVID, the differential diagnosis for gastrointestinal complaints is broad, with a high prevalence of noninfectious complications including nodular lymphoid hyperplasia, inflammatory bowel disease, atrophic gastritis, sprue-like illness with villous atrophy, and intestinal lymphangiectasia [10, 12].

Unlike in CVID, gastrointestinal disorders are relatively infrequently described in the XLA group, and their occurrence is not well known $[2,9,13$, 14]. The reported incidence of infectious diarrhea for patients with CVID and XLA varies from 5\% to approximately $30 \%$ [12]. Giardia lamblia is the most frequently identified pathogen in the stool of symptomatic patients.

Our observations seem to confirm the dominance of respiratory infections as a leading symptom among XLA individuals. In the analyzed group $73 \%$ of patients suffered from recurrent bronchitis and $59 \%$ from pneumonia. During immunoglobulin therapy the occurrence of LRTI was diminished [8]. Ear and sinus infections were observed in $41 \%$ and $34 \%$ of patients respectively. As many as 12 (27\%) patients had chronic lung disease, mostly bronchiectases. Seven of them developed it before diagnosis and immunoglobulin treatment, while 5 did so during therapy. CRS was observed in 7 (15.9\%) boys. In 3 of them it occurred on immunoglobulin therapy. Plebani [2] reported even much higher occurrence of CLD (24 of 73) and CRS (35 of 73) in an Italian group, indicating the risk of both complications during replacement immunoglobulin therapy. According to other reports, the incidence of ENT and airway infections in XLA patients is comparable $[11,12]$. Immunoglobulin replacement therapy significantly reduced the incidence of pneumonia, bronchitis and otitis [2, 15, 16]. Some data showed that IVIg was not protective enough in sinusitis and CLD in patients with CVID and XLA $[2,16]$. As also observed in our pa-

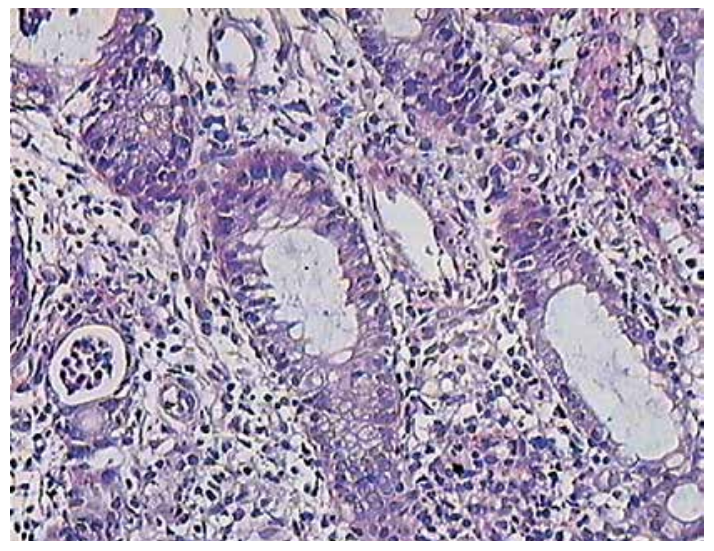

Figure 1. Colon biopsy in XLA patient with IBD: inflammatory infiltrates, neutrophils in the crypt epithelium, crypt abscess (on the left) H + E. 400x 
tients previously [8], after introduction of immunoglobulin replacement therapy, LRTI were seen less frequently. However, bronchiectases and sinusitis were recognized during the follow-up, regardless of immunoglobulin treatment.

Gastrointestinal disorders constitute a clinically important group of symptoms in PAD patients. About $10 \%$ of hypogammaglobulinemic patients suffer from unexplained severe diarrhea, abdominal pain, or wasting. In many patients with primary hypogammaglobulinemia diarrhea caused by G. lamblia, Campylobacter, Salmonella, or other microorganisms are reported. Gl disorders appear to be less frequent in XLA compared to CVID and other antibody deficiencies [9, 17-21]. The most common gastrointestinal symptom reported in XLA patients is chronic diarrhea, frequently related to G. lamblia, Salmonella, or Campylobacter, likely secondary to bacterial overgrowth and lack of antibody, with self-limitation when intravenous immunoglobulin therapy is introduced [22, 23].

In our group of Bruton's agammaglobulinemic patients the occurrence of gastrointestinal problems was high (63.6\%), mostly represented by chronic diarrhea (26 of 44 patients). The etiology in the majority of them remained unknown. In accordance with the findings of others, diarrhea was generally observed before diagnosis and before immunoglobulin replacement therapy was started. The majority of patients had the diarrhea controlled by immunoglobulins, and the problems disappeared on regular infusions. Surprisingly in view other observations, only in one boy was G. lamblia infection reported.

Among patients with primary antibody deficiency, especially those with CVID, inflammatory bowel disease and Crohn's disease are reported quite frequently [17, 24, 25]. Yet only incidental reports on Crohn's disease or Crohn's-like lesions in XLA patients can be found [7, 9]. Ament [26] reported 9 out of 39 patients with either XLA and CVID with colitis. In another study, Teahon et al. [27] presented 2 XLA and 10 CVID patients with mostly diarrhea not associated with known microbial pathogens, in whom intestinal inflammation unlike that found in classic IBD was observed. In 1 patient XLA treatment with an elemental diet was successful, while in the other jejunum resection was performed, revealing non-specific transmural inflammation. Cellier et al. [7] reported regional enteritis associated with enterovirus in an XLA patient, and rare incidence of chronic intestinal inflammation mimicking Crohn's disease in XLA patients. However it should be noted that in some large studies no evidence of inflammatory bowel disease (IBD) among agammaglobulinemic patients was reported, while gastrointestinal disorders were diagnosed in about $13 \%$ of patients $[2,19]$. Some authors indicate that
IBD/Crohn's disease, less common in XLA compared to CVID presumably, may be connected with T-cell dysfunction in the latter, which can cause hypogammaglobulinemia and different autoimmune phenomena, including cytopenia, granulomas and IBD [28]. Of the presented group, diagnosis of IBD was established incidentally, which confirmed the observations of other authors. In contrast to other reports, viral gastrointestinal infections within our patients were reported incidentally [3]. Only sporadic rotavirus or norovirus was identified in the analyzed group.

In conclusion, respiratory tract infections were the most common symptoms among the group of patients with XLA. Gastrointestinal disorders were one of the main manifestations of XLA, reported almost as often as LRTI. The most common Gl symptom was diarrhea, which usually resolved after immunoglobulin therapy was started. Infections caused by $G$. lamblia were reported occasionally. Inflammatory bowel disease was diagnosed quite exceptionally, which presumably may be connected with normal T cell immunity.

\section{Conflict of interest}

The authors declare no conflict of interest.

\section{References}

1. Smith CIE. X-linked agammaglobulinemia: a disease of BTK tyrosine kinase. In: Primary immunodeficiency diseases. $2^{\text {nd }}$ ed. Ochs H, Smith CIE, Puck J (eds.). Oxford Press, Oxford 2007.

2. Plebani A, Soresina A, Rondelli R, et al.; Italian Pediatric Group for XLA-AIEOP. Clinical, immunological and molecular analysis in a large cohort of patients with X-linked agammaglobulinemia: an Italian multicenter study. Clin Immunol 2002; 104: 221-30.

3. Aguilar C, Malphettes M, Donadieu J, et al. Prevention of infections during primary immunodeficiency. Clin Infect Dis 2014; 59: 1462-70.

4. Hernandez-Trujillo VP, Scalchunes C, Cunningham-Rundles C, et al. Autoimmunity and inflammation in X-linked agammaglobulinemia. J Clin Immunol 2014; 34: 627-32.

5. Howard V, Greene JM, Pahwa S, et al. The health status and quality of life of adults with $\mathrm{X}$-linked agammaglobulinemia. Clin Immunol 2006; 118: 201-8.

6. Agarwal S, Mayer L. Gastrointestinal manifestations in primary immune disorders. Inflamm Bowel Dis 2010; 16: 703-11.

7. Cellier C, Foray S, Hermine O. Regional enteritis associated with enterovirus in a patient with X-linked agammaglobulinemia. N Engl J Med 2000; 342: 1611-2.

8. Pac M, Bernatowska E. Polish experience with immunoglobulin replacement treatment by subcutaneous infusion. Centr Eur J Immunol 2005; 30: 346-50.

9. Conley ME, Howard V. Clinical findings leading to the diagnosis of X-linked agammaglobulinemia. J Pediatr 2002; 141: 566-71.

10. Fried AJ, Bonilla FA, Pathogenesis, diagnosis, and management of primary antibody deficiencies and infections. Microbiol Rev 2009; 22: 396-414. 
11. Driessen G, van der Burg M. Primary antibody deficiencies. Eur J Pediatr 2011; 170: 693-702.

12. Wood P, Stanworth S, Burton J, et al. Recognition, clinical diagnosis and management of patients with primary antibody deficiencies: a systematic review. Clin Exp Immunol 2007; 149: 410-23.

13. Etzioni A. Immune deficiency and autoimmunity. Autoimmun Rev 2003; 2: 364-9.

14. Agarwal S, Mayer L. Pathogenesis and treatment of gastrointestinal disease in antibody deficiency syndromes. J Allergy Clin Immunol 2009; 124: 658-64.

15. Ehlayel MS, Bener A, Laban MA. Primary immunodeficiency disease in children: 15 year experience in a tertiary care medical center in Qatar. J Clin 2013; 33: 317-24.

16. Quinti I, Soresina A, Spadaro G, et al. Long-term follow-up and outcome of a large cohort of patients with common variable immunodeficiency. J Clin Immunol 2007; 27: 308-16.

17. Daniels JA, Lederman HM, Maitra A, Montgomery EA. Gastrointestinal tract pathology in patients with common variable immunodeficiency (CVID). A clinicopathologic study and review. Am J Surg Pathol 2007; 12: 1800-12.

18. Washington K, Stenzel TT, Buckley RH, Gottfried RM. Gastrointestinal pathology in patients with common variable immunodeficiency and X-linked agammaglobulinemia. Am J Surg Pathol 1996; 20: 1240-52.

19. Chun JK, Lee TL, Song JW, Linton JA, Kim DS. Analysis of clinical presentation of Bruton disease: a review of 20 years of accumulated data from pediatric patients at Severance Hospital. Yonsei Med J 2008; 49: 28-36.

20. Zullo A, Romiti A, Rinaldi V, et al. Gastric pathology in patients with common variable immunodeficiency. Gut 1999; 45: 77-81.

21. Weber CR, Turner JR. Inflammatory bowel disease: is it really just another break in the wall? Gut 2007; 56: 6-8.

22. Glocker E, Grimbacher B. Inflammatory bowel disease: is it a primary immunodeficiency? Cell Mol Life Sci 2012; 69: 41-8.

23. Ariganello P, Angelino G, Scarselli A, et al. Relapsing Campylobacter jejuni systemic infections in a child with X-linked agammaglobulinemia. Case Rep Pediatr 2013; 2013: 735108.

24. Khodadad A, Aghamohammadi A, Parvaneh N, et al. Gastrointestinal manifestation in patients with common variable immunodeficiency. Dig Dis Sci 2007; 52: 2977-83.

25. Kalha I, Sellin JH. Common variable immunodeficiency and the gastrointestinal tract. Curr Gastroenterol Rep 2004; 6: 377-83.

26. Ament ME. Immunodeficiency syndromes and the gut. Scand J Gastroenterol 1985; 20 (Suppl. 114): 127-35.

27. Teahon K, Webster AD, Price AB, Weston J, Bjarnson I. Studies on the enteropathy associated with primary agammaglobulinemia. Gut 1994; 35: 1244-9.

28. Agarwal S, Mayer L. Diagnosis and treatment of gastrointestinal disorders in patients with primary immunodeficiency. Clin Gastroenterol Hepatol 2013; 11: 1050-63. 